\title{
Mechanical Valve Replacement for Congenital Heart Disease Complicated by Native Pulmonary Valve Endocarditis: A Case Report and Literature Review
}

\author{
Qing Wang, MD, Yang Gao, MD, Wang Xi, MD, Hua Shen, MD, Jing Wang, MD, Jian Xiao, MD, PhD, \\ Zhinong Wang, MD, PhD
}

Department of Cardiothoracic Surgery, Changzheng Hospital, Second Military Medical University, Shanghai, China

\section{ABSTRACT}

Congenital heart disease (CHD) is one of the most common risk factors for infective endocarditis. However, it is rare to find a CHD patient complicated by isolated pulmonary valve endocarditis. Here, we report an adult patient with congenital heart disease complicated by native pulmonary valve endocarditis who underwent a mechanical valve replacement. We also review previous literature to examine key points in the treatment of such patients.

\section{CASE REPORT}

Among all patients with congenital heart disease (CHD) complicated by infective endocarditis (IE), the right sided type constitutes only about 17.8\% [Loureiro-Amigo 2016]. Right sided endocarditis often involves tricuspid valve or prosthetic valve, and isolated native pulmonary valve endocarditis is rarely reported [Kang 2007]. Here, we report a woman with CHD complicated by native pulmonary valve endocarditis, who was given a successful mechanical valve replacement.

A 23-year-old woman was admitted to our hospital due to two months of chest pain, cough and fever without any apparent reason, such as tooth extraction or drug abuse. At admission, she presented with a temperature of $37.6^{\circ} \mathrm{C}$, a regular pulse of 101 beats per minute and a blood pressure of 92/58 mmHg. A loud systolic murmur (grade IV) could be heard at the left parasternal region, while there was no typical signs of IE, such as finger clubbing, Roth spots, splinter hemorrhages or Oslers nodes. Typical signs of heart failure or CHD, such as jugular vein distention, lower extremity edema, or cyanosis were not obvious.

A blood culture positive for Group A streptococci, sensitive to most antibiotics, was taken at admission. Transthoracic echocardiography (TTE) showed a large ventricular septal defect (Figure 1a), a narrow right ventricular outflow tract

Received fanuary 1, 2017; received in revised form December 1, 2017; accepted December 15, 2017.

Correspondence: Zhinong Wang, Department of Cardiothoracic Surgery, Changzheng Hospital, Second Military Medical University, Shanghai, 200003, China; +862181885901 (e-mail: wangzn007@163.com); Fian Xiao, Department of Cardiothoracic Surgery, Changzheng Hospital, Second Military Medical University, Shanghai, 200003, China; +862181885905 (email: 18916066266@189.cn).
(RVOT) (Figure 1b), pulmonary valve stenosis with a vegetation, moderate tricuspid valve regurgitation, and moderate pericardial effusion. Computed tomography angiography (CTA) (Figure 2a) confirmed the results of TTE. The diagnosis of CHD complicated by isolated pulmonary valve endocarditis was established. Two weeks after admission, her temperature was controlled and symptoms were greatly relieved after a comprehensive treatment, including use of Vancomycin.

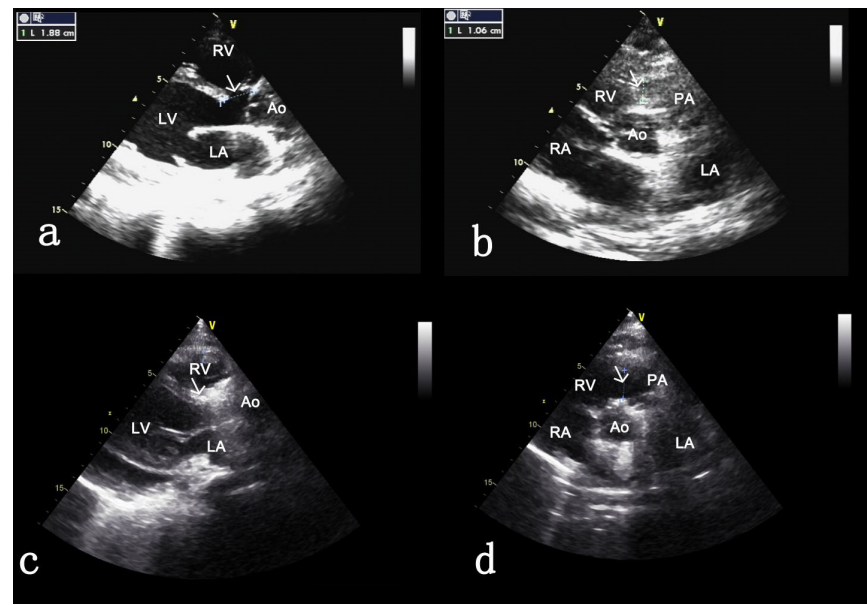

Figure 1. Transthoracic echocardiography showing the $\mathrm{CHD}$ before $(A, B)$ and after operation (C,D). A) Parasternal long axis view showing the presence of a large perimembranous VSD (indicated by white arrow); B) Aortic short axis view showing a narrow RVOT(indicated by white arrow); C) Repair of the VSD after the operation; D) Broadened RVOT after the operation. LA, left atrium; LV, left ventricle; RA, right atrium; RV, right ventricle; Ao, aorta; PA, pulmonary artery.

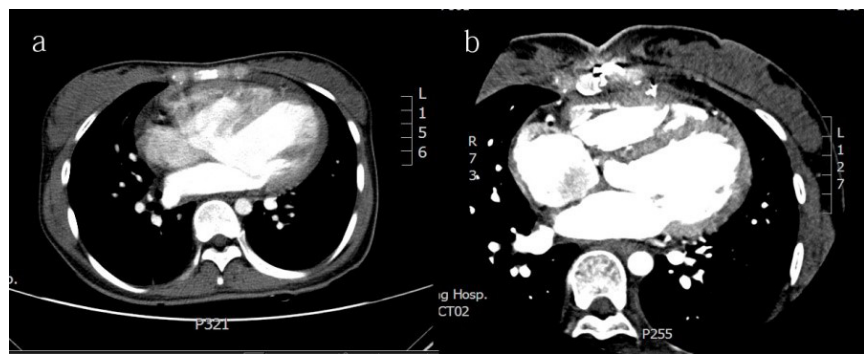

Figure 2. Computed Tomography Angiography revealing the VSD before $(A)$ and after the surgery $(B)$. 
Table 1. Previous reported cases of CHD complicated by pulmonary valve endocarditis.

\begin{tabular}{|c|c|c|c|c|c|c|c|c|}
\hline Lacalzada J et al & 2010 & 76 & male & PVS/ASD & No & No & Staph epidermidis & antibiotic therapy \\
\hline Bilen $\mathrm{E}$ et al & 2008 & 46 & male & $\mathrm{VSD} / \mathrm{RVH}$ & No & No & Not confirmed & surgery \\
\hline Chambers J et al & 1986 & 38 & female & VSD & No & No & Escherichia coli & antibiotic therapy \\
\hline Abdelbar $A$ et al & 2013 & 53 & male & RVOT obstruction & Yes & Pneumonia & Not confirmed & antibiotic therapy \\
\hline
\end{tabular}

Abbreviations: TOF, Tetralogy of Fallot; PVS, Pulmonary valve stenosis; VSD, Ventricular septal defect; ASD, Atrial septal defect; RVH, Right ventricular hypertrophy; RVOT, Right ventricular outflow tract; MRSA, Methicillin resistant staphylococcus aureus.

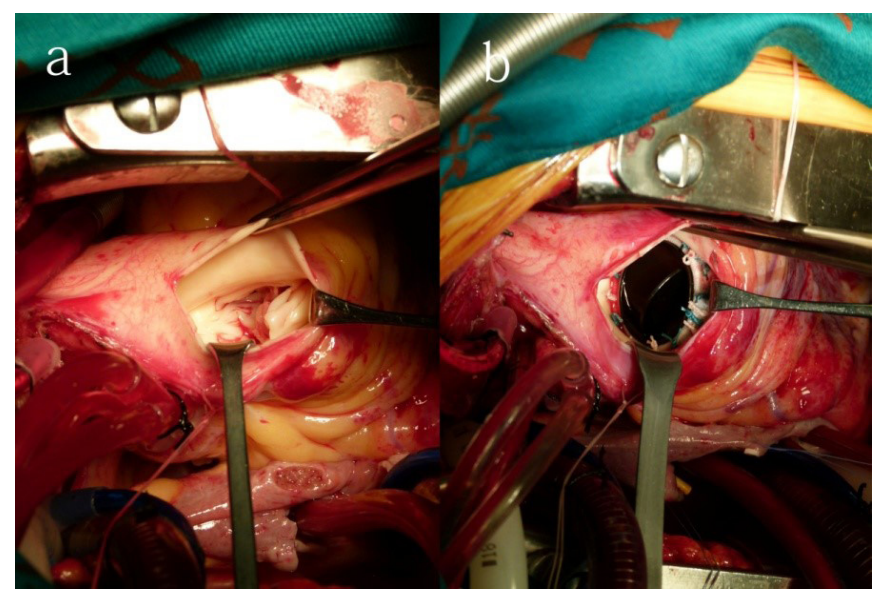

Figure 3. Photos in operation showing a dilated pulmonary aorta (A) and implantation of the mechanical pulmonary valve(B).

We conducted an operation of debridement of infective endocarditis, pulmonary valve replacement (PVR), ventricular septal defect repair, right ventricular outflow tract dredge and tricuspid annuloplasty under general anesthesia and hypothermic cardiopulmonary bypass. The patient was intubated with a single lumen endotracheal tube, and hemodynamics was monitored by a swan-ganz catheter. Following median careful re-sternotomy with a swing saw, aorto-bicaval cannulation was conducted to establish cardiopulmonary bypass (CPB). After the establishment of $\mathrm{CPB}$, the heart was opened. Verrucous vegetations were found on the pulmonary valve and debrided completely, and a CarboMedics $25 \mathrm{~mm}$ (A1-025) Futaba mechanical valve was chosen to replace the valve (Figure 3). A peripheral membranous ventricular septal defect of $2.0 \times 2.5 \mathrm{~cm} 2$ was probed and repaired with GoreTex patch. RVOT narrowed by fibrous annulus was dredged and resected. Mild prolapse with regurgitation of tricuspid leaflets was observed, and a concomitant tricuspid valvuloplasty was completed.

After the operation, the patient recovered uneventfully, and laboratory studies, TTE (Figure 1c, 1d), and a pulmonary

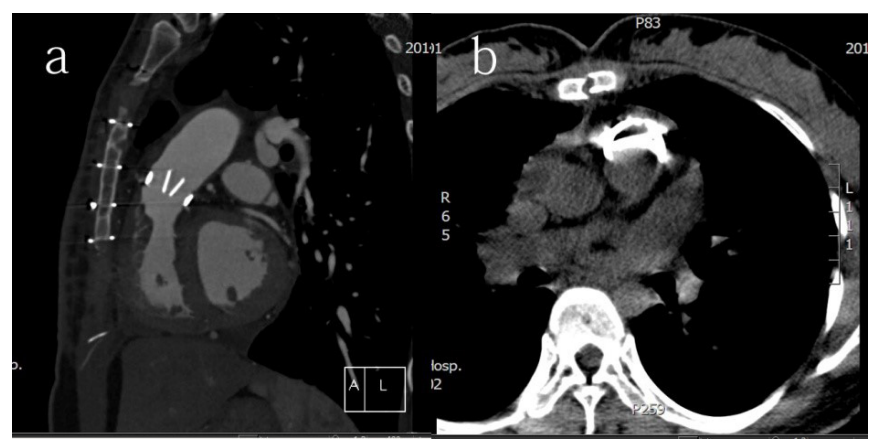

Figure 4. Computed Tomography showing a mechanical pulmonary valve replacement after the surgery in sagittal plane view (A) and cross section view (B).

CTA (Figure 4) were performed before her discharge and showed no abnormalities. A one year 3 month follow-up was conducted, which showed that the patient was in good condition without any symptoms. TTE and chest X-ray also showed no abnormalities.

\section{DISCUSSION}

The patient has been diagnosed with CHD since childhood, and we suspect that the abnormalities of hemodynamics caused by stenosis of the RVOT and dilation of the pulmonary artery contributed to the growth of vegetation. A review of the literature found 7 classic case reports of CHD complicated by pulmonary valve endocarditis (Table 1). However, there are no previous reports of a non-intravenous drug user with CHD complicated by isolated native pulmonary endocarditis and treated with a mechanical valve replacement, to our knowledge.

Besides the classic symptoms of endocarditis, such as fever, anemia weight loss and heart murmur, the right-sided endocarditis has peculiar respiratory symptoms, predisposing to misdiagnosis. In this case, the patient also had respiratory symptoms of cough, chest pain and dyspnea. Most patients in the cases we reviewed have respiratory symptoms of dyspnea, 
cough, and hemoptysis, etc., which are often caused by complications of pneumonia and pulmonary embolism, which may cause a misdiagnosis. Clinicians should consider IE and make routine physical examination for these patients. TTE is quite useful in differentiating those patients with pneumonia and other respiratory disease.

Because patients with right sided endocarditis often have a better prognosis than those with left sided endocarditis, clinicians tend to be more conservative with surgery for patients with pulmonary endocarditis. However, when conservative treatment is ineffective or patients have concomitant structural heart disease, it is necessary to conduct a surgery. Deng and colleagues [Deng 2013] reported 4 cases of pulmonary valve endocarditis undergoing surgical valve repair without replacement. PVR is less seen in clinics than other valve replacements because of less incidence of pulmonary endocarditis, better prognosis of pulmonary regurgitation, as well as the higher chance of embolism after PVR. During the operation, we found that the patient's pulmonary valve had been badly destroyed, which required a prosthetic replacement. Prostheses used in PVR at present can be classified into homograft valve-conduit, biological valve, and mechanical valve. These three types of prosthesis have their own pros and cons. According to Delahaye et al. [Delahaye 2014], patients undergoing bioprosthetics replacement have higher in-hospital and 1-year death rates, but his research was focused on bicuspid and aortic valves rather than the pulmonary valve. The author attributed this to patient characteristics rather than to valve dysfunction.

Considering economics, recurrence risk of IE, and the age of our patient, we made the decision to complete a mechanical valve replacement. In terms of mechanical PVR, anticoagulation therapy is of paramount importance. Sadeghpour et al. [Sadeghpour 2014] reported 38 patients who underwent mechanical PVR, and 7 of them (18\%) had malfunctioning pulmonary prostheses because of inadequate anticoagulation therapy. It is recommended that INR be controlled at approximately 3.0. We strictly controlled the INR at 2.8 and the follow-up results showed an absence of thromboembolism.

\section{CONCLUSION}

In conclusion, this report highlights three key points. First, even though pulmonary valve endocarditis is relatively rare clinically, physicians should not neglect the possibility of endocarditis when they encounter a patient with respiratory symptoms. Second, a mechanical valve can also be chosen in PVR. And finally, a more rigorous anticoagulation plan must be administered when a mechanical valve replacement is performed on the pulmonary valve.

\section{ACKNOWLEDGEMENTS}

This study was funded by Shanghai Leading Talent Program (LJRC-WZN) and Nature Science Foundation of Science and Technology Committee of Shanghai municipality (17ZR1439100).

\section{REFERENCES}

Bilen E, Yasar AS, Bilge M, et al. 2008. Isolated pulmonic valve endocarditis in an adult patient with ventricular septal defect and infundibular pulmonary stenosis. Echocardiography 25:904-7.

Delahaye F, Chu V H, Altclas J, et al. 2014. One-year outcome following biological or mechanical valve replacement for infective endocarditis. Int J Cardiol 178C:117-23.

Deng H, Ma Y, Zhai H, et al. 2013. Surgical valve repair of isolated pulmonary valve endocarditis. Interact Cardiovasc Thorac Surg 16:384-6.

Irving CA, Kelly D, Gould FK, et al. 2010. Successful medical treatment of bioprosthetic pulmonary valve endocarditis caused by methicillinresistant Staphylococcus aureus. Pediatr Cardiol 31:553-5.

Kang N, Smith W, Greaves, S, et al. 2007. Pulmonary-valve endocarditis. N Engl J Med 35:2224-5.

Lacalzada J, Enjuanes C, Izquierdo MM, et al. 2010. Pulmonary valve infective endocarditis in an adult patient with severe congenital pulmonary stenosis and ostium secundum atrial septal defect. Cardiol Res Pract 26:798956.

Loureiro-Amigo J, Fernández-Hidalgo N, Pijuan-Domènech A, et al. 2016. Infective endocarditis in adult patients with congenital heart disease: experience from a reference centre. Enferm Infecc Microbiol Clin 41:167-174.

Park HE, Cho GY, Kim HK, et al. 2009. Pulmonary valve endocarditis with septic pulmonary thromboembolism in a patient with ventricular septal defect. J Cardiovasc Ultrasound 17:138-40.

Sadeghpour A, Kyavar M, Javani B, et al. 2014. Mid-term outcome of mechanical pulmonary valve prostheses: the importance of anticoagulation. J Cardiovasc Thorac Res 6:163-8.

Sousa P, Santos W, Marques N, et al. 2013. A 72-year-old woman with an uncorrected tetralogy of Fallot presenting with possible pulmonary endocarditis: a case report. J Med Case Rep 2:150. 